\title{
Efficacy and safety of a novel personalized navigation template in proximal femoral corrective osteotomy for the treatment of $\mathrm{DDH}$
}

\author{
Qiang Shi ${ }^{1,2}$ and Deyi Sun ${ }^{1 *}$
}

\begin{abstract}
Background: This present study is aimed to retrospectively evaluate the efficacy and safety of a novel personalized navigation template in proximal femoral corrective osteotomy for the treatment of DDH.

Methods: Twenty-nine consecutive patients with DDH who underwent proximal femoral corrective osteotomy were evaluated between August 2013 and June 2017. Based on the different surgical methods, they were divided into the conventional group $(n=14)$ and navigation template group $(n=15)$. The osteotomy degrees, radiation exposure, and operation time were compared between the two groups.

Results: No major complications relating to osteotomy surgery such as redislocation or avascular necrosis occurred in the navigation template group, which had more accurate osteotomy degrees, less radiation exposure, and shorter operation time when compared with the conventional group $(P<0.05)$. Moreover, there was significant difference according to the McKay criteria between the two groups $(P=0.0362)$.

Conclusions: The novel personalized navigation template in proximal femoral corrective osteotomy is effective and safe, which could improve the femoral osteotomy accuracy, reduce radiation exposure, and shorten operation time.
\end{abstract}

Keywords: Femoral corrective osteotomy, 3D printing, Navigation template, Developmental dysplasia of the hip

\section{Background}

Developmental dysplasia of the hip (DDH) is considered one of the most common three-dimensional (3D) hip deformities in children [1]. Derotational femoral shortening osteotomy as part of the surgical procedure of DDH was firstly introduced by Klisic and Jancovic as an aid to decrease the complications related with surgery such as redislocation or avascular necrosis [2]. Other authors have also regarded proximal femoral corrective osteotomy as an effective procedure for treating DDH

\footnotetext{
* Correspondence: sun950092@Hotmail.com

1 Department of Sports Medicine, Xiangya Hospital, Central South University, Changsha 410008, People's Republic of China

Full list of author information is available at the end of the article
}

[3-5]. The performance and positioning of femoral osteotomy is one of the most important surgical procedures to ensure ideal correction effects, but the conventional radiographic imaging methods, such as $\mathrm{x}$-ray or $\mathrm{CT}$ (computed tomography), were not sufficient to provide accurate information about complex deformities [6]. Moreover, individual differences in the size and shape of femur remain changeable factors that may essentially influence the accuracy of femoral osteotomy.

Zheng et al. [7] designed a 3D-printed navigation template for the proximal femoral varus rotation and shortening osteotomy. However, it was doubtful using the template to correct angles of femoral anteversion and neck-shaft simultaneously due to different planes. Based 
on these concerns, the present study introduces a novel design and application of derotational femoral shortening osteotomy with navigation template for the treatment of DDH in children. Therefore, this study was performed to assess the efficacy and safety of navigation template for more accurate osteotomy degrees, less radiation exposure, and shorter operation time when compared with the conventional operation, thus to result in a clinically viable alternative which is simple and precise.

\section{Materials and methods \\ Patients}

From August 2013 to June 2017, 29 consecutive patients (7 males and 22 females) presenting DDH who underwent derotational femoral shortening osteotomy were included in our hospital. Patients with subluxation of hip or classified as grade I were not involved in this study. Based on different surgical methods, they were divided into the conventional group $(n=14)$ and navigation template group $(n=15)$. There was no significant difference between the two groups in terms of age, gender, side, Tönnis classification, and surgery history (Table 1). This study was approved by the Ethics Review Committee of Xiangya Hospital Central South University, and all parents in the present study signed an informed consent to participate.

\section{Digital design and navigation template preparation}

Preoperatively, all patients underwent $\mathrm{x}$-rays and femoral $\mathrm{CT}$ scanning. The obtained slice CT scanning data in navigation template group were saved as DICOM (Digital Imaging and Communications in Medicine) format and then transferred into the Mimics 20.0 software

Table 1 Comparison of demographic data and deformity characteristics between two groups

\begin{tabular}{llll}
\hline Characteristics & $\begin{array}{l}\text { Conventional } \\
\text { group }(n=14)\end{array}$ & $\begin{array}{l}\text { Navigation template } \\
\text { group }(n=15)\end{array}$ & $P$ value \\
\hline Mean age (range), years & $4.2 \pm 1.6(3-8)$ & $3.7 \pm 2.2(2-8)$ & 0.478 \\
Gender, $n$ (\%) & & & 0.742 \\
Male & $3(21.4)$ & $4(26.7)$ & \\
Female & $11(78.6)$ & $11(73.3)$ & 0.597 \\
Side, $n$ (\%) & & & \\
Left & $8(57.1)$ & $10(66.7)$ & 0.657 \\
Right & $6(42.9)$ & $5(33.3)$ & \\
Tönnis classification & & & \\
II & $4(57.1)$ & $3(20.0)$ & \\
III & $7(54.5)$ & $10(66.7)$ & \\
IV & $3(18.2)$ & $2(13.3)$ & \\
Surgery history & & & \\
Yes & $8(57.1)$ & $7(46.7)$ & \\
No & $6(42.9)$ & $8(53.3)$ & \\
\hline
\end{tabular}

(Materialise, Leuven, Belgium) for three-dimensional reconstruction. 3D models of femur were accurately reconstructed and exported in STL (Standard Tessellation Language) format. Thereafter, the deformed 3D models were imported into the UG 6.0 software. The osteotomy plane and accurate planning for the derotational degrees were defined based on the femoral parameters (Fig. 1a). And then, the personalized corrective osteotomy template model with four guide holes was fitted to the bone surface with reference to the characteristic configuration of the deformed femur and anatomical landmarks (Fig. 1b). The designed corrective osteotomy template was then printed using 3D printing technology with materials of acrylic resin.

3D reconstruction was performed using the Amira 3.1 software. By tracking the contours of the prominent bone structure for reconstruction, adjusting the geometric alignment of the overlapping point contours, modeling the surface by meshing into a polygonal point frame, and reconstructing the target contour based on the point data. The result is a fully interactive $3 \mathrm{D}$ visualization of the reconstructed structure based on radiology and CT imaging data, allowing full visualization of the area. The 3D models were saved in STL format for stereolithography, which is a universal international file standard for 3D modeling and printing, and then imported into the Imageware (version 12.0; EDS, Palo Alto, CA) software for further analysis. The optimal osteotomy plane and accurate planning for the derotational degrees were defined based on the femoral parameters using 3D printing technology. System parameters included the thickness of the processing layer at $0.1 \mathrm{~mm}$, processing speed at $500 \mathrm{~mm} / \mathrm{s}$. The entire process of prototype construction required approximately $5-18 \mathrm{~h}$, with an average of $8.9 \mathrm{~h}$.

\section{Surgical procedures}

All the proximal femoral corrective osteotomies in the present study were performed by one senior orthopedic surgeon in our department. The navigation template was sterilized and applied intraoperatively to assist derotational femoral shortening osteotomy based on preoperative simulation (Fig. 2). When the proximal femur was exposed, usually via a separate lateral incision, the navigation template was matched to the proximal lateral femur in the best possible manner (Fig. 3a). Four K-wires were inserted through the navigation holes of the template (Fig. 3b), then the bone was osteotomized through a slit on the template. The template was then removed with the K-wires left in place, matching with the shortening osteotomy bone which was required to provide a pressure-free reduction. Rotating the K-wires into the parallel status and this position was maintained with a reduction guide (Fig. 3c). Therefore, we were able to perform all osteotomies as preoperatively simulated. 

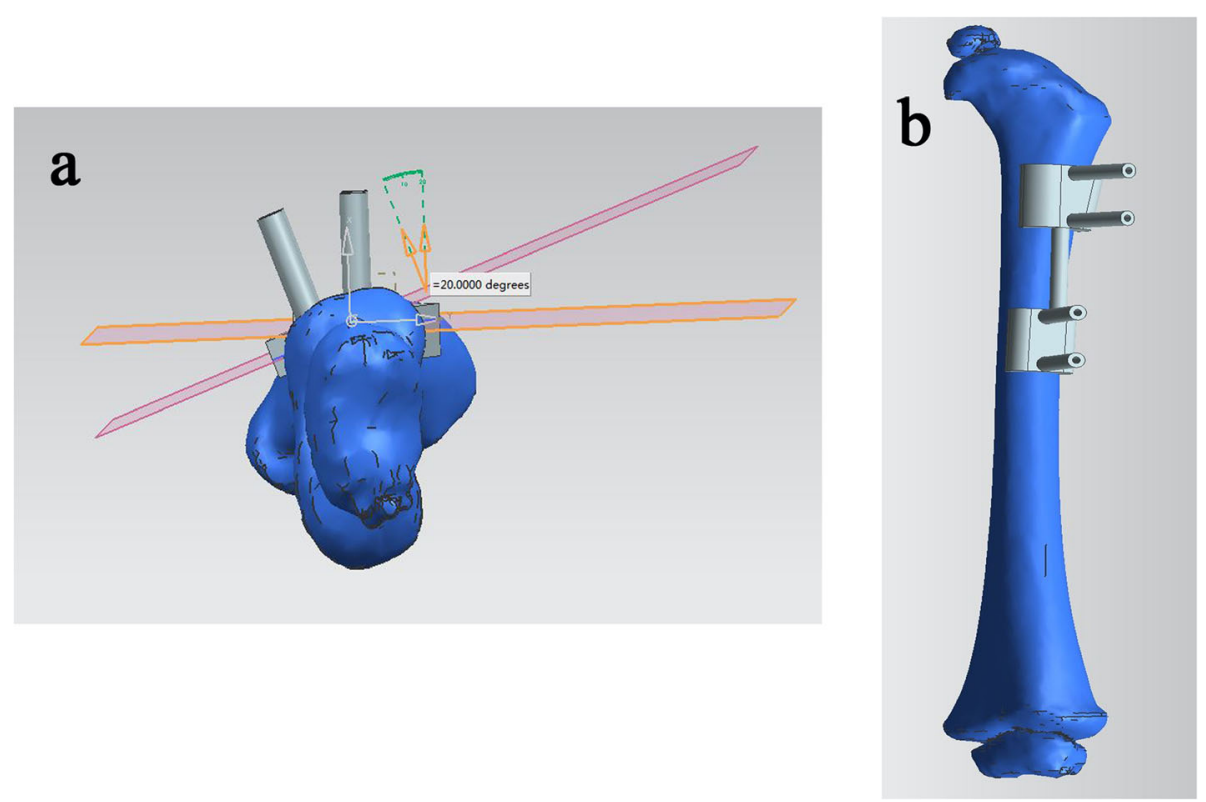

Fig. 1 Design of osteotomy angle and plane. a The osteotomy plane and accurate planning for the degree of derotation were defined based on the femoral parameters. $\mathbf{b}$ Simulation of the navigational template on the femur

Finally, they were managed by internal fixation with Locking Compression Plate (LCP) (Fig. 3d). On the other hand, the derotational degrees of the osteotomy and shortening distance were performed on the basis of preoperative and intraoperative measurements in the conventional group.

\section{Postoperative management}

No significant difference in postoperative management procedures after derotational femoral shortening osteotomy between the two groups. A spica cast fixation was used for 8 weeks and double lower limb brace with hip abduction for another 8 weeks. Weight bearing was
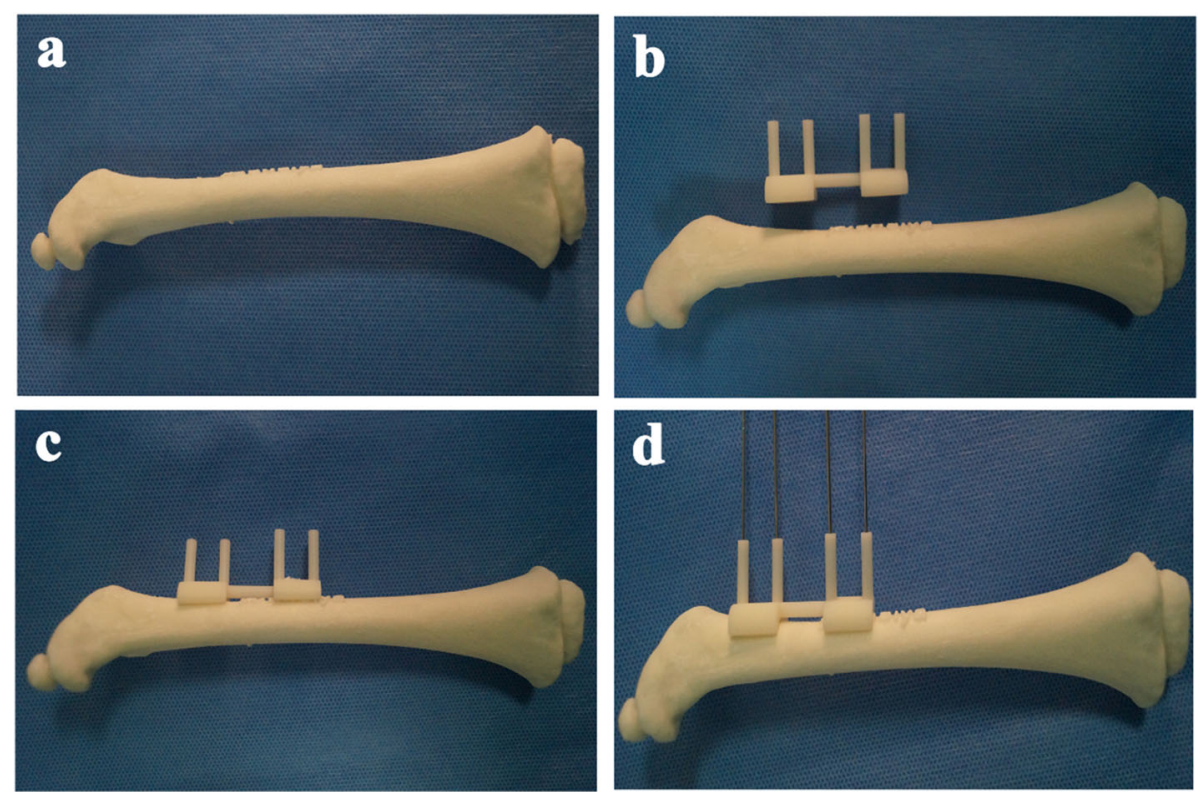

Fig. 2 Simulated operation with 3D printing model and navigation template. a 3D printing model of the femur. b 3D printing model of the femur and navigational template. c The navigational template fitted 3D printing model of femur perfectly. $\mathbf{d}$ The K-wires was inserted through the navigational template 

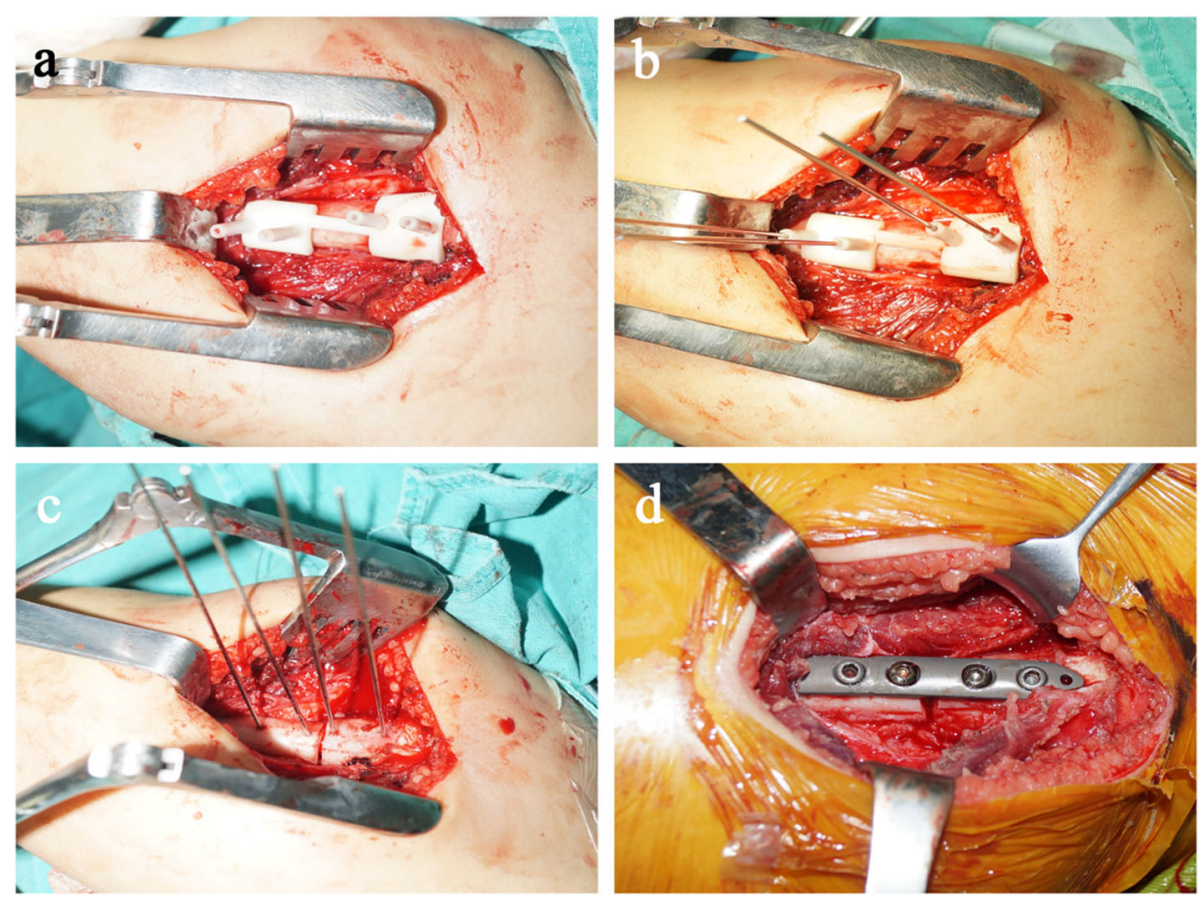

Fig. 3 Intraoperative operation using the navigation template. a The navigation template fitted the surface of the femoral bone perfectly. $\mathbf{b}$ Four K-wires were inserted applying the template. $\mathbf{c}$ The template was removed, and a reduction guide was used to maintain the K-wire in a parallel position. $\mathbf{d}$ Internal fixation was then accomplished with LCP

guided to start after removal of the brace. Moreover, radiographs of the pelvis and femur were taken regularly until the region of osteotomy completely healed, and then the internal fixation was removed. The mean follow-up was 4.8 years ( 3 to 7 years), and patients were reviewed at 8 weeks, 4 and 12 months, and then every year until skeletal maturity.

\section{Statistical analysis}

Quantitative data in this study were statistically analyzed by the SPSS 25.0 software (SPSS, Inc., Chicago, USA) and manifested as count (percentage) or mean \pm standard deviation (SD). Student's $t$ test, chi-squared test, and Fisher's exact test were applied to analyze the data in this study. Different parameters measured between two groups were evaluated with independent $t$ test for continuous variables and chi-square test or Fisher's exact test for the categorical variables. A $P$ value $<0.05$ was considered statistically significant.

\section{Results}

There was statistical significance in osteotomy degrees $(P=0.0003)$, radiation exposure $(P<0.0001)$, and operation time $(P<0.0001)$ when compared with the conventional group (Table 2). Regarding the osteotomy degrees, there was statistical significance between the conventional group $\left(23.6 \pm 7.3^{\circ}\right)$ and navigation template group (18.3 $\left.\pm 5.4^{\circ}, P=0.0003\right)$. However, as for the shortening length, there was no statistical significance between the conventional group $(2.3 \pm 0.7 \mathrm{~cm})$ and navigation template group $(2.4 \pm 0.3 \mathrm{~cm}, P=0.1149)$. The operation time in the navigation template group $(20.6 \pm$ $3.9 \mathrm{~min}$ ) was significantly less than that of the conventional group $(37.3 \pm 8.8 \mathrm{~min}, P<0.0001)$. Based on the McKay criteria, 13 patients in the navigation template group obtained excellent correction, 1 patient obtained good prognosis, and 1 patient obtained fair prognosis, while 5 patients were scored as excellent, 5 as good, 1 as fair, and 1 as poor in the conventional group. As a

Table 2 Comparison of operation data and functional outcomes between two groups

\begin{tabular}{llll}
\hline & $\begin{array}{l}\text { Conventional } \\
\text { group }(n=14)\end{array}$ & $\begin{array}{l}\text { Navigation template } \\
\text { group }(n=15)\end{array}$ & $P$ value \\
\hline Osteotomy degrees, $^{\circ}$ & $23.6 \pm 7.3$ & $18.3 \pm 5.4$ & 0.0003 \\
Shortening length, cm & $2.3 \pm 0.7$ & $2.4 \pm 0.3$ & 0.1149 \\
$\begin{array}{l}\text { Radiation exposure, } \\
\text { times }\end{array}$ & $8.1 \pm 2.8$ & $3.0 \pm 1.4$ & $<0.0001$ \\
$\begin{array}{l}\text { Operation time, min } \\
\text { McKay standard, } n(\%)\end{array}$ & $37.3 \pm 8.8$ & $20.6 \pm 3.9$ & $<0.0001$ \\
Excellent & $5(35.7)$ & $13(86.7)$ & 0.0362 \\
Good & $5(35.7)$ & $1(6.7)$ & \\
Fair & $2(14.3)$ & $1(6.7)$ & \\
Poor & $2(14.3)$ & 0 & \\
\hline
\end{tabular}


consequence, the surgical outcome between the two groups was no significant difference $(P=0.0362)$.

Clinical assessment including measurement of leglength inequality, hip range of motion, and gait pattern showed normal motion and gait. No patient had significant limb shortening, and no major complications relating to osteotomy surgery such as redislocation or avascular necrosis occurred in the navigation template group after 18 months of follow-up.

\section{Typical case}

A 2-year-old girl who was found limping by her parents came to our department, and radiographs of the pelvis showed that her left femoral head was dislocated from the normal position (Fig. 4a). According to the Tönnis classification, this girl was classified as grade III. Assisted by $3 \mathrm{D}$ navigation template, derotational femoral shortening osteotomy was performed, and the proximal femur was fixed at $20^{\circ}$ of derotation with LCP (Fig. $4 \mathrm{~b}$ ). The bone healing of the femoral osteotomy was achieved 4 months after surgery. After 18 months of follow-up, normal motion and gait with leg-length equality were found, and the anteversion angle of the left femur was $17^{\circ}$, which was evaluated as excellent according to the McKay criteria [8].

\section{Discussion}

Accurate operative procedures of DDH are technically challenging for surgeons. It is well accepted that derotational femoral shortening osteotomy is always necessary to facilitate the open reduction of $\mathrm{DDH}$, especially in high dislocations and older children, correct abnormal femoral anteversion angle, reduce excessive pressure on the femoral head, and decrease the intraoperative instability of the hip [9]. In the guide-pins method, one pin was inserted into proximal and the other distal to the osteotomy position $[10,11]$. When the distal pin lies parallel to the proximal pin, the desired derotation of the femur was achieved. The rate for overcorrection of affected legs was $44 \%$ and $15 \%$ for undercorrection, respectively. In the present study, we achieved satisfactory results in 15 cases by using 3D navigational template.

In order to complete the operation safely and achieve the desired effect of DDH, it is crucial to acquire as much deformity information in various situations as possible. Radiography, 2D, or 3D CT images alone cannot provide sufficiently accurate information about complex hip deformities [12, 13]. Size and shape differences in the proximal femur also affect the accuracy of osteotomy. Furthermore, due to limited exposure of lateral cortex of the proximal femur, performing derotational femoral shortening osteotomy accurately remains challenging [14]. Computer-aided navigation is an effective and useful tool to improve the accuracy of operation $[15,16]$. However, they are expensive equipment, timeconsuming procedures, and have a significant learning curve.

Recently, many 3D navigation templates have been introduced in the trauma, joint, and spine fields [17-19], but only a few researchers have reported their applications in the pediatric orthopedic disorders. Previously, different personalized templates have been designed for cubitus varus deformity and achieved wonderful clinical outcome [20-22]. Lu et al. [23] developed a novel patient-specific template in congenital scoliosis and validated the accuracy and safety. More recently, Otsuki et al. [24] constructed 3D custom cutting guide for curved peri-acetabular osteotomy. With its accurate guide, this template was considered to avoid serious complications associated with osteotomy. Compared with other templates, the present study had applied a novel personalized navigation template in proximal femoral corrective osteotomy for the treatment of DDH and validated the efficacy and safety in clinical settings.
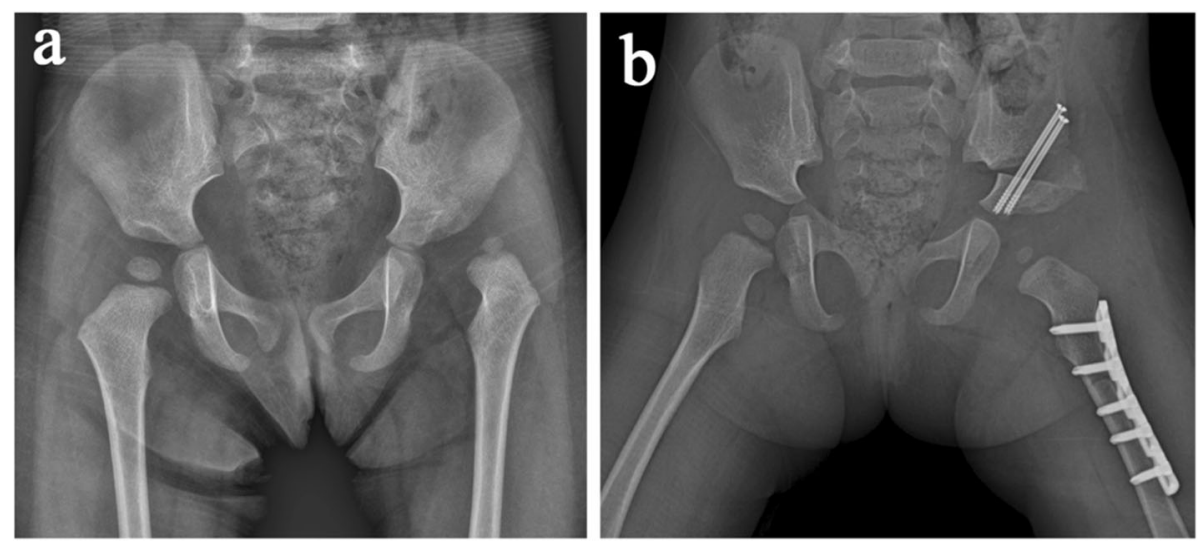

Fig. 4 Radiographs before and after the operation. a Preoperative anteroposterior radiograph of left DDH in the navigation template group. b Postoperative radiograph showed anatomical correction and good appearance 1 week after surgery 
Neither redislocation nor avascular necrosis was detected in the navigation template group, which had more accurate osteotomy degrees, less radiation exposure, and shorter operation time when compared with the conventional group $(P<0.05)$. Moreover, there was significant difference according to the McKay criteria between the two groups $(P=0.0362)$.

The advantage of the $3 \mathrm{D}$ navigation template is that it is simple and easy to apply which can be performed without much expertise and special training. Moreover, the template can be used during surgery to assist surgical navigation and accurate osteotomy. Lastly, we can check the accuracy of individual template with the aid of $3 \mathrm{D}$ printing model before surgery, thereby eliminating the need for repeated manipulates and shortening the surgical time. Besides, fewer radiographs are needed, and continual fluoroscopic monitoring can be avoided intraoperatively.

Nevertheless, because the $3 \mathrm{D}$ navigation template is developed based on a preliminary series, it has several limitations in the present study. On the one hand, muscle and fat tissue on the bone can affect the position of the template. So, an exact preparation of the bone surface was essential, and the template should fit tightly to the corresponding lateral femur to allow accurate drill trajectories. Furthermore, more cases and multi-center prospective studies in the future are needed to evaluate the accuracy and efficacy of the 3D navigation template. In addition, it is necessary to further prolong the followup time because the patients in our study were still at the stage of skeletal immaturity.

\section{Conclusion}

The application of a novel personalized navigation template for derotational femoral shortening osteotomy can simplify the operation and generate a highly accurate and reliable correction. Compared with the traditional freehand technique, which requires a long learning curve and experienced surgeons, the use of our 3D navigation template has the advantages of more accurate osteotomy degrees, less radiation exposure, and shorter operation time.

\section{Abbreviations}

3D: Three-dimensional; CT: Computed tomography; DDH: Developmental dysplasia of hip; DICOM: Digital imaging and communications in medicine; STL: Standard Tessellation Language; LCP: Locking Compression Plate

\section{Acknowledgements}

Not applicable.

\section{Authors' contributions}

DS is the guarantor of the integrity of the entire study. QS wrote the manuscript. DS performed the femoral osteotomy surgery. DS and QS contributed to the study concepts/design, the data acquisition, the drafting and critical revision of the manuscript, the approval of the final manuscript, and the manuscript editing. QS did the literature research. Both authors read and approved the final manuscript.

\section{Funding}

No benefits in any form have been received or will be received from a commercial party related directly or indirectly to the subject of this article.

\section{Availability of data and materials}

The data used to support the findings of this study are available from the corresponding author upon request.

\section{Ethics approval and consent to participate}

This study was approved by the institutional review board of the Xiangya Hospital Central South University. All patients gave written informed consent before participating in this study.

\section{Consent for publication}

All patients and their families gave their written informed consent for the publication of their individual data and identifying photographs.

\section{Competing interests}

The authors declare that they have no competing interests.

\section{Author details}

'Department of Sports Medicine, Xiangya Hospital, Central South University, Changsha 410008, People's Republic of China. ${ }^{2}$ Key Laboratory of Organ Injury, Aging and Regenerative Medicine of Hunan Province, Changsha 410008, People's Republic of China.

Received: 8 May 2020 Accepted: 30 July 2020

Published online: 12 August 2020

\section{References}

1. Sewell MD, Eastwood DM. Screening and treatment in developmental dysplasia of the hip-where do we go from here? Int Orthop. 2011;35(9): 1359-67.

2. Klisic P, Jankovic L. Combined procedure of open reduction and shortening of the femur in treatment of congenital dislocation of the hips in older children. Clin Orthop Relat Res. 1976;119:60-9.

3. Sankar WN, Neubuerger CO, Moseley CF. Femoral anteversion in developmental dysplasia of the hip. J Pediatr Orthop. 2009;29(8):885-8.

4. Sankar WN, Tang EY, Moseley CF. Predictors of the need for femoral shortening osteotomy during open treatment of developmental dislocation of the hip. J Pediatr Orthop. 2009;29(8):868-71.

5. Wenger DR, Lee CS, Kolman B. Derotational femoral shortening for developmental dislocation of the hip: special indications and results in the child younger than 2 years. J Pediatr Orthop. 1995;15(6):768-79.

6. Turker M, Cirpar M, Cetik O, Senyucel C, Tekdemir I, Yalcinozan M. Comparison of two techniques in achieving planned correction angles in femoral subtrochanteric derotation osteotomy. J Pediatr Orthop B. 2012; 21(3):215-9

7. Zheng P, Xu P, Yao Q, Tang K, Lou Y. 3D-printed navigation template in proximal femoral osteotomy for older children with developmental dysplasia of the hip. Sci Rep. 2017;7:44993.

8. McKay DW. A comparison of the innominate and the pericapsular osteotomy in the treatment of congenital dislocation of the hip. Clin Orthop Relat Res. 1974;98:124-32

9. Wedge $\mathrm{JH}$, Kelley SP. Strategies to improve outcomes from operative childhood management of DDH. Orthop Clin North Am. 2012;43(3):291-9.

10. Dreher T, Wolf S, Braatz F, Patikas D, Doderlein L. Internal rotation gait in spastic diplegia--critical considerations for the femoral derotation osteotomy. Gait Posture. 2007;26(1):25-31.

11. Kay RM, Rethlefsen SA, Hale JM, Skaggs DL, Tolo VT. Comparison of proximal and distal rotational femoral osteotomy in children with cerebral palsy. J Pediatr Orthop. 2003;23(2):150-4.

12. Facanha-Filho FA, Winter RB, Lonstein JE, Koop S, Novacheck T, L'Heureux EA Jr, et al. Measurement accuracy in congenital scoliosis. J Bone Joint Surg Am. 2001;83(1):42-5.

13. Mao K, Wang Y, Xiao S, Liu Z, Zhang Y, Zhang $X$, et al. Clinical application of computer-designed polystyrene models in complex severe spinal deformities: a pilot study. Eur Spine J. 2010;19(5):797-802.

14. Hung SS, Lee ZL, Lee MY. Clinical application of rapid prototype model in pediatric proximal femoral corrective osteotomy. Orthopedics. 2008;31(1):72. 
15. Akiyama H, Goto K, So K, Nakamura T. Computed tomography-based navigation for curved periacetabular osteotomy. J Orthop Sci. 2010;15(6): 829-33.

16. Hsieh $\mathrm{PH}$, Chang $\mathrm{YH}$, Shih $\mathrm{CH}$. Image-guided periacetabular osteotomy: computer-assisted navigation compared with the conventional technique: a randomized study of 36 patients followed for 2 years. Acta Orthop. 2006; 77(4):591-7.

17. Li B, Lei $P$, Liu H, Tian X, Wen T, Hu R, et al. Clinical value of 3D printing guide plate in core decompression plus porous bioceramics rod placement for the treatment of early osteonecrosis of the femoral head. J Orthop Surg Res. 2018;13(1):130.

18. Li YT, Hung CC, Chou YC, Chen JE, Wu CC, Shen HC, et al. Surgical treatment for posterior dislocation of hip combined with acetabular fractures using preoperative virtual simulation and three-dimensional printing model-assisted precontoured plate fixation techniques. Biomed Res Int. 2019;2019:3971571.

19. Zheng $P$, Yao Q, Xu P, Wang L. Application of computer-aided design and 3D-printed navigation template in Locking Compression Pediatric Hip Plate(TauMu) placement for pediatric hip disease. Int J Comput Assist Radiol Surg. 2017;12(5):865-71.

20. Zhang YZ, Lu S, Chen B, Zhao JM, Liu R, Pei GX. Application of computeraided design osteotomy template for treatment of cubitus varus deformity in teenagers: a pilot study. J Shoulder Elbow Surg. 2011;20(1):51-6.

21. Murase T, Oka K, Moritomo H, Goto A, Yoshikawa H, Sugamoto K. Threedimensional corrective osteotomy of malunited fractures of the upper extremity with use of a computer simulation system. J Bone Joint Surg Am. 2008;90(11):2375-89.

22. Takeyasu Y, Oka K, Miyake J, Kataoka T, Moritomo H, Murase T. Preoperative, computer simulation-based, three-dimensional corrective osteotomy for cubitus varus deformity with use of a custom-designed surgical device. J Bone Joint Surg Am. 2013;95(22):e173.

23. Lu S, Zhang YZ, Wang Z, Shi JH, Chen YB, Xu XM, et al. Accuracy and efficacy of thoracic pedicle screws in scoliosis with patient-specific drill template. Med Biol Eng Comput. 2012;50(7):751-8.

24. Otsuki B, Takemoto M, Kawanabe K, Awa Y, Akiyama H, Fujibayashi S, et al. Developing a novel custom cutting guide for curved peri-acetabular osteotomy. Int Orthop. 2013;37(6):1033-8.

\section{Publisher's Note}

Springer Nature remains neutral with regard to jurisdictional claims in published maps and institutional affiliations.

Ready to submit your research? Choose BMC and benefit from:

- fast, convenient online submission

- thorough peer review by experienced researchers in your field

- rapid publication on acceptance

- support for research data, including large and complex data types

- gold Open Access which fosters wider collaboration and increased citations

- maximum visibility for your research: over $100 \mathrm{M}$ website views per year

At $\mathrm{BMC}$, research is always in progress.

Learn more biomedcentral.com/submissions 